\title{
New horizons towards thermalization
}

\author{
Ideas from theorists in fields as disparate as quantum gravity, quantum information and many-body localization are \\ finding common ground, as we explore in this month's Focus issue on quantum thermalization.
}

T

he thermalization of systems into a state of equilibrium is a phenomenon central to our understanding of physics, and statistical physics in particular. It washes out a system's memory of its initial state, maximizes entropy and underpins what has become an axiom of physics in the form of the second law of thermodynamics. Quantum systems are no exception: as long as they satisfy the ergodic hypothesis, their unitary evolution will lead them to thermalize ${ }^{1}$.

Over the decades, two types of system have garnered particular attention for their unusual behaviour on their path to thermalization: the first concerns chaotic systems - of which black holes are an example - where information stored in the original state is erased very quickly, resulting in fast equilibration. The other is known as many-body localization (MBL), in which thermalization fails to occur and information about the initial state is maintained, at least partially.

The trail leading to the understanding of these two types of system began with speculation - a familiar course for theoretical physics. For MBL, for example, the journey began when Philip Anderson asked whether certain closed random system may fail to thermalize ${ }^{2}$. To explore this question, he considered systems comprising a huge number of degrees of freedom. Although he was able to find an answer in the non-interacting case, leading to the concept of Anderson localization, the existence of localization in a many-body system - where even more complicated multiparticle configurations are involved remained elusive.

The study of chaotic systems, on the other hand, is often connected to black holes. These are arguably the fastest information scramblers we know of, because any information entering the black hole becomes irretrievable. However, as has been amply publicized, a formidable challenge arises when attempting to reconcile the properties and behaviour of black holes with quantum mechanics. This poses difficulties to the study of quantum chaos - even simply in coming up with a quantum model that captures the essential chaotic characteristics of a black hole. The contradictions seem to be fundamental. Gerard 't Hooft ${ }^{3}$ and Leonard Susskind ${ }^{4}$ have argued that to solve these essential issues one must revisit the very structure of space and time. And although they provided an avenue towards a possible solution, referred to as quantum holography, many crucial details remain to be sorted out.

As with other physical theories that begin as blurry ideas, the way to approach these two limiting regimes of thermalization has become clearer with the help of advances in both the theoretical machinery and experimental insights available to researchers. Tools such as the renormalization group and tensor networks have greatly improved our capability of handling many-body problems. The discovery of anti-de Sitter space (AdS)/conformal field theory (CFT) correspondence lays the ground for building a theory of quantum gravity that satisfies the holographic principle ${ }^{5}$, thus shedding light on the solution to the black hole information paradox. Moreover, a new quantitative tool known as the out-oftime-order-correlator (OTOC) provides an appealing measure of the effective loss of local quantum information during the thermalization process itself. This quantum information perspective may therefore lead to new insights into the dynamics of MBL and chaotic states.

In this month's Focus, we provide an overview of recent developments along three different but interconnected research strands, showcasing the diversity of approaches that are contributing to this research field.

In a Perspective, Ehud Altman surveys the progress that has been made in unravelling the physics of MBL, including the existence of MBL states in different dimensions, and some dynamical phenomena that characterize MBL as a new distinct phase of matter. An example is the existence in the MBL phase of a nonequilibrium state of matter known as a time crystal ${ }^{6,7}$.

In another Perspective, Xiao-Liang Qi focuses on recent developments in quantum gravity, using the AdS/CFT duality as the main prism for our understanding. Roughly speaking, the duality provides a one-to-one correspondence between two theories: one describes systems living in higher spacetime dimensions (the bulk), and the other for those in lower dimensions (the boundary). It has recently been realized that concepts from quantum information theory such as entanglement and quantum error correction may enter the above description of spacetime in a fundamental way, implying that there is a deep connection between many-body states and spacetime geometry.

Finally, Brian Swingle gives an introduction to OTOC and its wider implications in a third Perspective. Providing a quantitative way to study the dynamics of quantum information, and then probe thermalization or lack thereof, this approach may help us to understand MBL and the black hole information problem. Recently, OTOC has been measured in experiments using nuclear magnetic resonance ${ }^{8}$ and trapped ions ${ }^{9}$, thus bringing it from a thought experiment into the laboratory. This is nontrivial as it requires control over a quantum system exquisite enough to enable precise reversal of its time evolution. These experimental successes will no doubt encourage further measurements of OTOC in more complex quantum systems in the near future.

Although it seems increasingly clear that these three topics are related to each other, it is also remarkable to note their vastly different energy scales to which they pertain. As with so many examples in the history of physics, progress made in one field may lead to breakthroughs in another. Researchers working on different problems may find themselves surveying a horizon much vaster then they ever could have anticipated.

Published online: 3 October 2018 https://doi.org/10.1038/s41567-018-0326-2

\footnotetext{
References

1. Deutsch, J. M. Phys. Rev. A 43, 2406-2409 (1991).

2. Anderson, P. W. Phys. Rev. 109, 1492-1505 (1958).

3. 't Hooft, G. Preprint at https://arxiv.org/abs/gr-qc/9310026 (1993).

4. Susskind, L. J. Math. Phys. 36, 6377-6396 (1995).

5. Maldacena, J. Int. J. Theor. Phys. 38, 1113-1133 (1999).

6. Zhang, J. et al. Nature 543, 217-220 (2017).

7. Choi, S. et al. Nature 543, 221-225 (2017).

8. Li, J. et al. Phys. Rev. X 7, 031011 (2017).

9. Gärttner, M. et al. Nat. Phys. 13, 781-786 (2017).
} 